\title{
PREGNANCY IN A RUDIMENTARY HORN OF BICORNUATE UTERUS
}

\author{
Lt Col JK GOEL ${ }^{*}$, Lt Col M CHANDRASHEKHAR ${ }^{+}$
}

MJAFI 2000; 56 : 351-352

KEY WORDS : Ectopic pregnancy; Rudimentary horn

\section{Introduction}

$\mathbf{E}$ ctopic pregnancy was first recognized by Busiere in 1663 on examining the body of an executed prisoner in Paris. Gifford made a more complete report in 1731 in England, describing the condition in which the fertilised ovum was implanted anywhere outside the uterine cavity [1]. During this decade the number of hospitalisations for ectopic pregnancy has tripled and ectopic pregnancy has emerged as a leading cause of maternal morbidity $[2,3]$. Fortunately the risk of death has declined despite increasing incidence of disease. The incidence of ectopic pregnancy in non-white patients is approx $12 / 1000$. Out of all ectopic pregnancies, $98.5 \%$ are singleton tubal pregnancies and rest is at other sites, which create diagnostic problems and have been associated with greater morbidity and mortality than tubal pregnancies. Pregnancy in the rudimentary horn of a bicornuate uterus is a very rare condition and Johnsen reported the incidence of this preginancy at approx. one in one lakh pregnancies [4]. The case is being reported because of its rarity.

\section{Case Report}

18 year old lady was admitted on 10 Nov 1998 at 1230 hrs with history of amenorrhoea of 14 weeks duration and pain left iniac fossa since 0930 hrs. She had one vomiting, gave history of giddiness. She denied history of any vaginal bleeding/urinary/bowel problems.

Past menstrual cycles had been 3-4/1-2 months, irregular with average flow and without any dysmenorrhoea, LMP-05 Aug 98. Obstetric History - Married for one year had been staying with husband for last six months.

On Examination - There was no pallor, no tachycardia, and blood pressure was $100 / 60 \mathrm{~mm}$ of $\mathrm{Hg}$. Per Abdomen Examination - revealed tenderness in supra pubic region and left iliac fossa however no palpable mass/guarding was present. Pelvic examination showed uterus of parous size and pushed to the right. There was a palpable mass of about 12 weeks size pregnant uterus, soft, mobile and acutely tender on the left. Cervical rocking was positive and there was brownish staining on the examining finger. Haemogram done was within normal limits. With a provisional diagnosis of cornual pregnancy left, as urgent ultra sonography was asked for, which also showed an un-ruptured comual ectopic pregnancy.

Emergency exploratory laparotomy done showed uterus slightly enlarged with right tube and ovary normal. The gestational sac was seen bulging from the superior surface of left rudimentary horn of uterus. Left tube and ovary were apparently normal. However, attachment of left round ligament was unusual and confusing, as it was attached medially to the gestational sac on the main uterus just below the pedicle of rudimentary horn. Rudimentary horn along with gestational sac was removed. Histopathological examination confirmed the gestational sac in the myometrium of the rudimentary horn of the uterus. Patient made an uneventful postoperative recovery and was discharged to home on the 10th day.

\section{Discussion}

The clinical diagnosis of ectopic pregnancy is not at all straight forward.The "classic" clinical triad of pain, abnormal bleeding and palpable adnexal mass is not commonly present and when present, may erroneously lead to diagnosis of ectopic pregnancy.

This pregnancy commonly presents with abdominal pain, which may occur before or after rupture. Diagnosis of rudimentary horn pregnancy is usually made at laparotomy [5]. In this rare form of ectopic preg-

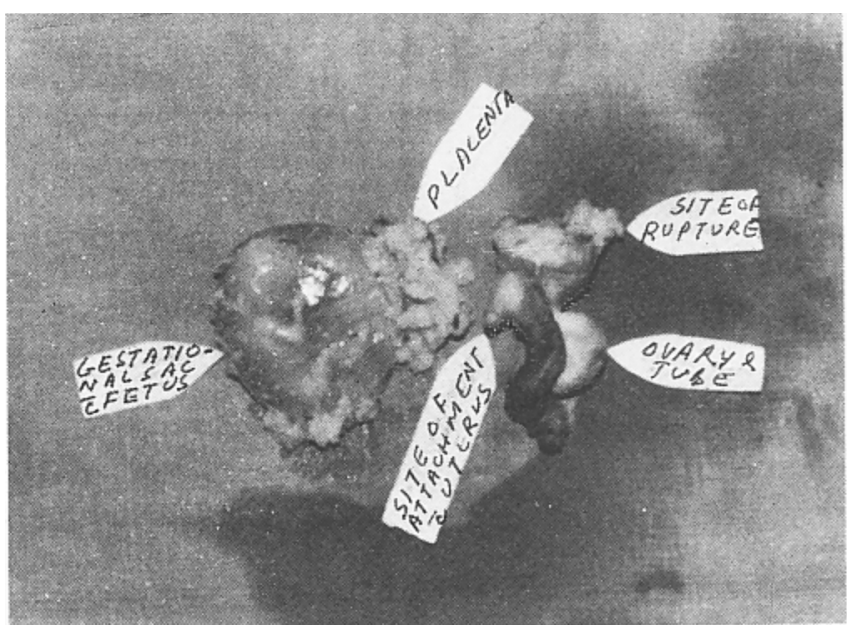

Fig. 1 : Showing foetus in a gestational sac with placenta and site of rupture in rudimentary horn of uterus. Left ovary and tube is also seen

"Classified Specialist (Obstetrics \& Gynaecology), ${ }^{+}$Classified Specialist (Pathology), Military Hospital, Belgaum - 09. 
nancy implantation occurs in the cavity of rudimentary horn of uterus. The horn does not always communicate with the rest of uterine cavity in which case it must be assumed that sperms ascend through the other horn of uterus and get fertilized with an ovum in the peritoneal cavity. This, then enters the tube of rudimentary horn. An important feature of this pregnancy is that the sac is surrounded by myometrium and even though this is poorly developed, it contains the pregnancy for a longer time than in tube/ovary [6]. Rupture does not usually take place until 12 to 20 weeks but when it does, there is always severe bleeding which makes the condition extremely dangerous. By the end of II trimester, $89 \%$ of these pregnancies had ruptured as reported by O'Leary [7]. This pregnancy is associated with a mortality rate as high as 5\% [4]. Removal of the gravid rudimentary horn and leaving behind normal horn of uterus along with tube and ovary is the accepted treatment. Where removal of ru. dimentary horn is not possible, a total hysterectomy has to be performed [8].

\section{References}

1. Rock JA, Thompson JD, Te Linde's Operative Gynaecology : Ectopic Pregnancy, Eighth edition. Philadelphia : J.B. Lippincott-Raven, 1997;501-27. .

2. Dorfman SF, Deaths from Ectopic Pregnancy, United States, 1979-80 Obster Gynaecol 1983;62;334-42.

3. Weinsten L, Marris MB, Dotters D, Christian CD. Ectopic Pregnancy-A new surgical epidemic 1983;61:698-701.

4. Johnsen K. Pregnancy in a rudimentary horn, two case reports Obstet Gynaecol 1969;34:805-8.

5. Remaine BB. Non tubal ectopic pregnancy Clinjcal Obstet Gynaecol 1987;30:191-9.

6. Chawla CD, Saxena RK, Murlidhar C. Rupture of Gravid hom of bicornuate Uterus. MJAFI 1995;51:138-9.

7. O'Leary JL. O'Leary JA. Rudimentary hom pregnancy. Obstet Gynaecol 1963;22;371-6.

8. Mysercough PR, Munrokerr's Operative Obstertics 10th edition New York Mac Millan Publishing Co Inc 1996;371-99 